\title{
GRIN3A and MAPT stimulate nerve overgrowth in macrodactyly
}

\author{
$\mathrm{XU} \mathrm{SHI}^{1^{*}}, \mathrm{LU} \mathrm{LU}^{2 *}, \mathrm{XIU} \mathrm{JIN}^{3}, \mathrm{BIN} \mathrm{LIU}^{4}, \mathrm{XIGUANG} \mathrm{SUN}^{4}$, LAIJIN LU $^{4}$ and YANFANG JIANG ${ }^{1,5}$ \\ ${ }^{1}$ Department of Genetic Diagnosis Center, Central Laboratory; Departments of ${ }^{2}$ Breast Surgery, ${ }^{3}$ Burn Surgery and \\ ${ }^{4}$ Hand and Foot Surgery, The First Hospital of Jilin University, Changchun, Jilin 130000; ${ }^{5}$ Key Laboratory of Zoonosis Research, \\ Ministry of Education, Institute of Zoonosis, Jilin University, Changchun, Jilin 130000, P.R. China
}

Received September 30, 2015; Accepted October 12, 2016

DOI: $10.3892 / \mathrm{mmr} .2016 .5923$

\begin{abstract}
As an uncommon and congenital condition, macrodactyly is characterized by an increase in the size of all the elements or structures of the digits or toes; however, the underlying pathogenesis remains to be fully elucidated. In the present study, the gene expression profiles of abnormal nerves were examined in three patients with macrodactyly using microarray analysis to identify potential genes contributing to nerve overgrowth. Gene expression profiling in the nerve tissue samples were scanned using the microarray and the differentially expressed genes were verified at the transcription level using reverse transcription-quantitative polymerase chain reaction analysis. Western blot analysis was used to determine the expression of target genes at the translational level. To confirm the upregulated genes during the process of nerve proliferation, SH-SY5Y cells were induced to differentiate into a neuronal-like phenotype using retinoic acid. A total of 165 genes showed significant changes ( $\geq 5$-fold) in gene expression, which may be associated with the development of limbs in macrodactyly. Glutamate ionotropic receptor NMDA 3A (GRIN3A) and microtubule-associated protein tau (MAPT) were identified as important contributors in promoting nerve overgrowth. Furthermore, it was identified that GRIN3A and $M A P T$ were regulated by the cAMP-protein kinase A and extracellular signal-regulated kinase $1 / 2$ pathways, respectively. The identification of genes expressed at high levels in macrodactyly may reveal potential factors, which contribute
\end{abstract}

Correspondence to: Professor Yanfang Jiang, Department of Genetic Diagnosis Center, Central Laboratory, The First Hospital of Jilin University, 71 Xinmin Street, Changchun, Jilin 130000, P.R. China

E-mail: yanfangjiang@hotmail.com

Professor Laijin Lu, Department of Hand and Foot Surgery, The First Hospital of Jilin University, 71 Xinmin Street, Changchun, Jilin 130000, China.

E-mail: vlulaijin@hotmail.com

*Contributed equally

Key words: macrodactyly, nerve overgrowth, gene expression, microarray to abnormal nerve proliferation and underpin the pathogenesis of macrodactyly, and provide potential application targets in nerve tissue regeneration engineering.

\section{Introduction}

Macrodactyly is an uncommon congenital condition characterized by an increase in the size of all the elements or structures of the digits or toes, including phalanges, tendons, vessels, subcutaneous fat and finger nails. The malformation often occurs unilaterally or asymmetrically and affects more than one digit or toe. The clinical conditions associated with this deformity are carpal tunnel syndrome, syndactylism, neurofibromatosis type 1 cafe au lait spots, lipoma and nevi (1-3).

The malformations in the fingers or toes are considered to be associated with other syndromes of macrodactyly, including vascular malformations, multiple enchondromatosis, maffuci syndrome, tuberous sclerosis and neurofibromatosis type $1(4,5)$. The exact pathogenesis underlying this condition and, in particular, the role of nerve growth stimulators in macrodactyly, remains to be fully elucidated. The present study aimed to determine nerve overgrowth stimuli using gene expression profiling was performed in malformed enlarged nerve tissues from the digits or toes of patients with macrodactyly. A total of six overexpressed (>10-fold) genes, including creatine kinase, mitochondrial 2 (CKMT2), vasoactive intestinal peptide $(V I P)$, FXYD domain-containing ion transport regulator 3 ( $F X Y D 3$ ), Glutamate ionotropic receptor NMDA 3A (GRIN3A), GSTT1 and microtubule-associated protein tau $(M A P T)$ were identified for their potential contribution to abnormal nerve overgrowth. In addition, MAPT and GRIN3A were identified as key regulators of nerve outgrowth. These nerve growth stimulators may contribute to nerve regeneration and reconstruction following nerve injury.

\section{Materials and methods}

Patients. The present study was reviewed and approved by the Ethics Committee of the First Hospital of Jilin University, (Changchun, China). Three male patients between the ages of 17 and 25 were recruited from the First Hospital of Jilin University between May 2011 and September 2013. All enrolled patients underwent surgical management for isolated nonsyndromic macrodactyly. Normal nerve tissue samples, which served as healthy controls, were obtained from five 
Table I. Primer sequences used in reverse transcription-polymerase chain reaction analysis.

\begin{tabular}{llll}
\hline Gene & Accession no. & \multicolumn{1}{c}{ Forward primer } & \multicolumn{1}{c}{ Reverse primer } \\
\hline CKMT2 & NM_001825.2 & 5'-GCCGCAATGCTTCTCTG-3' & 5'-GGCCATCCCAGCACAT-3' \\
VIP & NM_003381.3 & 5'-TCCTTGTGCTCCTGACTC-3' & 5'-CTGCTCCTCTTTCCATTC -3' \\
FXYD3 & NM_005971.3 & 5'-GTGACCCTGGGCCTGCT-3' & 5'-CAGCTTTGGGCTGAGCCT-3' \\
GRIN3A & NM_133445.2 & 5'-GGAGGTAGATGATGAAGGC-3' & 5'-AAACAAGAGGGCATAACAG-3' \\
GSTT1 & NM_000853.2 & 5'-ATCTTTGCCAAGAAGAACG-3' & 5'-TGTGAGGACCAGTAAGGAAG-3' \\
MAPT & NM_016835.4 & 5'-GCCAAAGGGCAGGATG-3' & 5'-TTCGGGAAGTGACAGAAGAG-3' \\
GAPDH & NM_002046 & 5'-GGAGTCAACGGATTTGGTC-3' & 5'-CCCCAGCCTTCTCCAT-3'
\end{tabular}

CKMT2, creatine kinase, mitochondrial 2; VIP, vasoactive intestinal peptide; FXYD3, FXYD domain-containing ion transport regulator 3; GRIN3A, glutamate ionotropic receptor NMDA 3A; GSTT1, glutathione S-transferase $\theta 1 ; M A P T$, microtubule-associated protein tau.

patients undergoing elective surgery for unrelated reasons, including road traffic accidents or fires. Written informed consent was obtained from guardians on the behalf of all participants.

Tissue treatment and reverse transcription-quantitative polymerase chain reaction $(R T-q P C R)$ analysis. A sample $(\sim 10 \mathrm{mg})$ of enlarged nerve tissue from a digit or toe was obtained from each of the patients with macrodactyly, which were sectioned into smaller sections $\left(\sim 3 \mathrm{~mm}^{3}\right)$, snap frozen in liquid nitrogen and stored at $-80^{\circ} \mathrm{C}$ until further use. The frozen tissues were homogenized in cold normal saline $(0.9 \%$ $\mathrm{NaCl}$ ), using a ULTRA-TURRAX Tube Drive Workstation (IKA Werke GmbH \& Co. KG, Staufen, Germany). Next, RNA was extracted from the nerve tissue samples using TRIzol (Invitrogen; Thermo Fisher Scientific, Inc., Waltham, MA USA) according to the manufacturer's protocol. A total of 500 ng RNA was used for reverse transcription was performed using the GoScript Reverse Transcription system (Promega Corporation, Madison, WI, USA). Next, the products were amplified using Power SYBR Green Master Mix (containing SYBR Green I Dye, AmpliTaq Gold® DNA Polymerase, dNTPs, passive reference and optimized buffer) on an ABI 7300 (Applied Biosystems; Thermo Fisher Scientific, Inc.). RT-qPCR was performed with an initial $10 \mathrm{~min}$ at $95^{\circ} \mathrm{C}$ followed by 40 cycles of $95^{\circ} \mathrm{C}$ for $15 \mathrm{sec}$, and $60^{\circ} \mathrm{C}$ for $1 \mathrm{~min}$. All experiments were repeated three times. Relative gene expression was calculated with the $2^{-\Delta \Delta \mathrm{Cq}}$ method (6) following normalization to the expression of GAPDH. The primers used for PCR are listed in Table I.

Cell culture. The SH-SY5Y cell line was obtained from the Cell Bank of the Chinese Academy of Sciences (Shanghai, China), and cultured in Dulbecco's modified Eagle's medium supplemented with $10 \%$ fetal bovine serum (all from Gibco; Thermo Fisher Scientific, Inc.) and $1 \%$ penicillin/streptomycin (Sigma-Aldrich; Merck Millipore, Darmstadt, Germany) at $37^{\circ} \mathrm{C}$ with $5 \% \mathrm{CO}_{2}$. The cells were sub-cultured when they reached $85-90 \%$ confluence and then seeded at a density of $5 \times 10^{3}$ cells $/ \mathrm{cm}^{2}$ into 12 -well plates (Nest Scientific USA, Rahway, NJ, USA) for $24 \mathrm{~h}$ at $37^{\circ} \mathrm{C}$. The cells were then induced to differentiate into neuronal cells by treating the cells with $10 \mu \mathrm{M}$ retinoic acid for $72 \mathrm{~h}$ at $37^{\circ} \mathrm{C}$ (7). Subsequently, the differentiated cells were treated with protein kinase A (PKA) inhibitor H89 or extracellular signal-regulated kinase (ERK)1/2 inhibitor PD98059 (Beyotime Institute of Biotechnology, Haimen, China) for up to $4 \mathrm{~h}$ at $37^{\circ} \mathrm{C}$.

Western blot analysis. The cells were collected and treated with lysis buffer (Beyotime Institute of Biotechnology) supplemented with $1 \%$ protease inhibitor mixture (Sigma-Aldrich; Merck Millipore). Subsequently, cell lysates were centrifuged at $10,000 \times \mathrm{g}$ for $15 \mathrm{~min}$ at $4^{\circ} \mathrm{C}$. Protein quantification was performed using BCA Protein Quantification kit (Thermo Fisher Scientific, Inc.) and $30 \mu \mathrm{g}$ protein per lane were separated on $10 \%$ SDS PAGE gel and then transferred onto PVDF membranes (Invitrogen; Thermo Fisher Scientific, Inc.). The membranes were incubated with $5 \%$ skimmed dry milk for $30 \mathrm{~min}$ and washed three times with TBST (10 mM Tris, $150 \mathrm{mM} \mathrm{NaCl}$ and $0.1 \%$ Tween-20). Next, the membranes were incubated with rabbit anti-human GRIN3A polyclonal antibody (cat. no. sc-98986; 1:5,000) and rabbit anti-human MAPT polyclonal antibody (cat. no. sc-32828; $1: 1,000$ ) at $4^{\circ} \mathrm{C}$ overnight. Then the goat anti-rabbit IgG-HRP secondary antibody (cat. no. sc-2302; 1:1,000) was used to incubate the membranes for $2 \mathrm{~h}$ at $37^{\circ} \mathrm{C}$. All antibodies were purchased from Santa Cruz Biotechnology, Inc. (Dallas, TX, USA). Densitometry scores were determined using Quantity One software, version 4.6.9 (Bio-Rad Laboratories, Inc., CA, USA).

Microarray. The RNA from the nerve tissue samples was hybridized and scanned using the Agilent Microarray Scanner (Agilent Technologies, Inc., Santa Clara, CA, USA) at the National Engineering Center for Biochip (Shanghai, China), according to the manufacturer's protocol. The raw data were obtained using Feature Extraction 10.7 software (Agilent Technologies, Inc.) with default settings and normalized using the Quantile algorithm in Gene Spring 11.0 software (Agilent Technologies, Inc.).

Spring 11.0 software. Gene Ontology (GO) functional annotation clustering analysis was used to process the microarray data. The GO project database (geneontology.org/) was used following the criteria of the Database for Annotation, Visualization and Integrated Discovery (david.abcc.ncifcrf.gov/) 
Table II. Downregulated genes ( $>10$-fold) in the macrodactyly tissue samples.

\begin{tabular}{lll}
\hline Gene symbol & Fold change & \\
\hline$A B L 2$ & 0.0901 & Description \\
CCL20 & 0.0571 & Chemotactic factor; associated with inflammation \\
CDC14B & 0.0523 & Serine/threonine phosphatase; dephosphorylate p53 \\
LAMA1 & 0.0674 & $\alpha$ subunit of laminin \\
$I L 1 B$ & 0.0740 & Inflammatory cytokine \\
LIF & 0.0613 & Inhibits embryonic stem cell differentiation; promotes germ cell proliferation \\
MBP & 0.0945 & Myelin sheath component \\
MFSD2 & 0.0872 & Mediates syncytin-2-dependent cell-cell fusion \\
NR4A1 & 0.0764 & Nuclear transcriptional activator \\
OSM & 0.0558 & Inhibits tumor proliferation; regulates cytokines \\
$P L A U R$ & 0.0825 & Degrades the extracellular matrix \\
SPATA13 & 0.0836 & Activates Rho-associated pathway; spermatogenesis-associated \\
$S O D 2$ & 0.0947 & Superoxide dismutase; \\
$S O X 11$ & 0.0828 & Antigen in B lymphomas; tumor suppressor \\
\hline
\end{tabular}

$A B L 2$, ALB proto-oncogene 2, non-receptor tyrosine kinase; CCL20, chemokine (C-C motif) ligand 20; $C D C 14 B$, cell division cycle 14 B; $L A M A 1$, laminin subunit $\alpha 1 ; I L 1 B$, interleukin $1 \beta ; L I F$, leukemia inhibitory factor; $M B P$, myelin basic protein; $M F S D 2$, major facilitator superfamily domain-containing 2; NR4A1, nuclear receptor subfamily 4, group A, member 1; OSM, oncostatin M; PLAUR, plasminogen activator, urokinase receptor; SPATA13, spermatogenesis-associated 13; $S O D$, superoxide dismutase.

Table III. Upregulated genes ( $>10$-fold) in the macrodactyly tissue samples.

\begin{tabular}{lcl}
\hline Gene symbol & Fold change & Description \\
\hline CKMT2 & 10.4 & Creatine kinase \\
VIP & 12.6 & Neuropeptide, belongs to a glucagon/secretin superfamily \\
FXYD3 & 11.2 & Na, K-ATPase ion channel regulator \\
GRIN3A & 10.8 & Ionotropic glutamate receptor \\
GSTT1 & 13.4 & Catalyzes the conjugation of reduced glutathione to electrophilic \\
& & moeities of xenobiotics or endogenous compounds \\
MAPT & 12.7 & Microtubule-associated protein tau \\
\hline
\end{tabular}

CKMT2, creatine kinase, mitochondrial 2; VIP, vasoactive intestinal peptide; FXYD3, FXYD domain-containing ion transport regulator 3; GRIN3A, glutamate ionotropic receptor NMDA 3A; GSTT1, glutathione S-transferase 01 ; MAPT, microtubule-associated protein tau.

for grouping genes according with the relevant biological processes, molecular functions and cellular component categories.

Statistical analysis. Statistical analyses were performed using GraphPad software, version 5.0 (GraphPad, Inc., La Jolla, CA, USA). All data are expressed as the mean \pm standard deviation. Student's $t$-test was performed to analyze the results of the gene expression profiling assays. $\mathrm{P}<0.05$ was considered to indicate a statistically significant difference.

\section{Results}

Gene expression profile analysis in macrodactyly. The present study first examined the gene expression profile in nerve tissue samples from three patients with macrodactyly. Of the 29,378 genes analyzed, 22 genes were upregulated ( $\geq 5$-fold) and 143 genes were downregulated ( $\geq 5$-fold) in the macrodactyly samples, compared with genes in the normal control samples (Fig. 1A). In particular, 14 genes were downregulated (Table II) and six genes were upregulated $>10$-fold in the macrodactyly samples (Table III). The genes upregulated in the samples from the patients with macrodactyly were considered as possible targets, which may promote nerve overgrowth.

The differentially expressed genes were grouped according to their biological process using Gene Ontology annotation (Fig. 1B). In the patients with macrodactyly, the majority of the differentially expressed genes were involved in metabolic process $(27.5 \%)$, response to stimulus $(20.1 \%)$ and immune system process $(16.9 \%)$.

Confirmation of genes, which may contribute to nerve overgrowth. The upregulated genes identified in the present study ( $\geq 10$-fold) may promote nerve overgrowth, therefore, these 
A

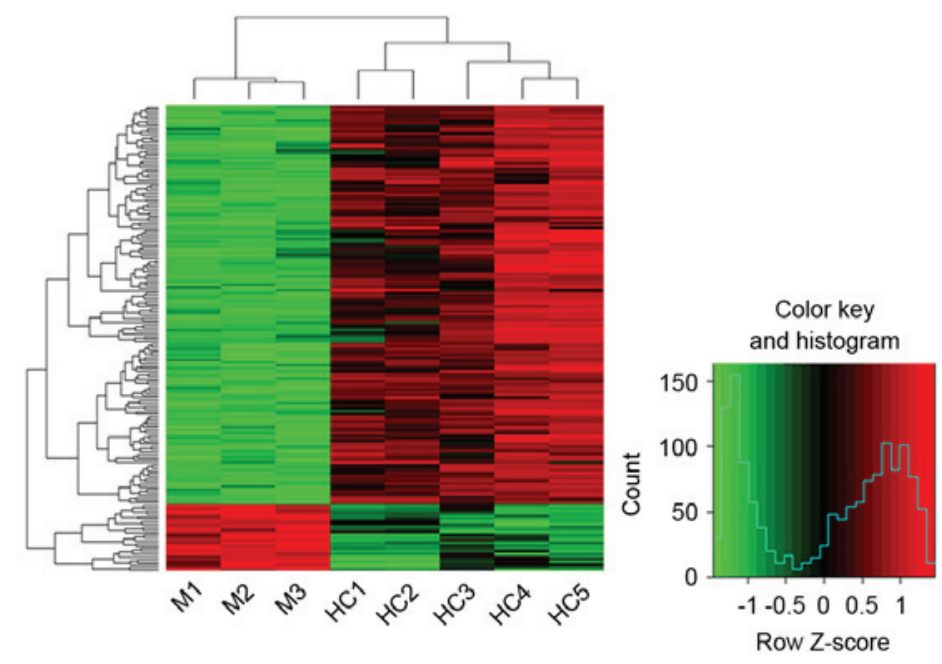

B

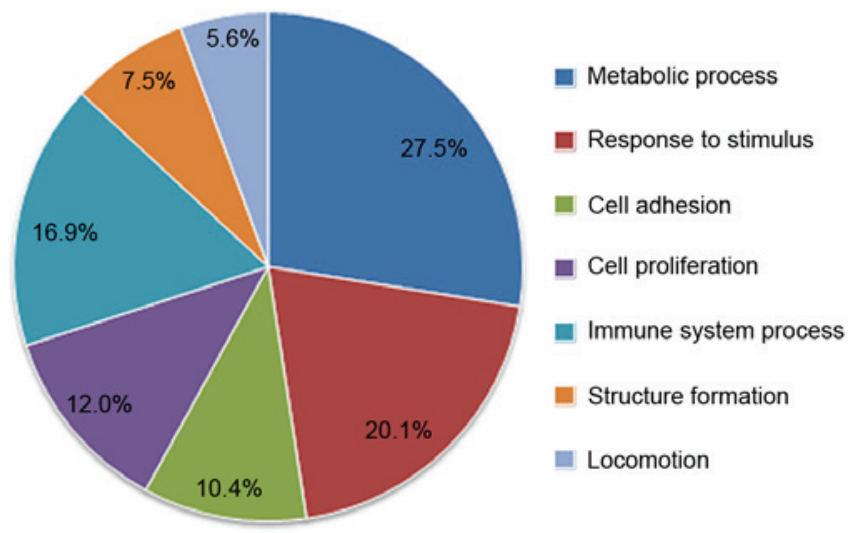

Figure 1. Gene expression profile analysis. (A) Hierarchical clustering of differentially expressed genes in macrodactyly. Dendrogram indicates gene expression profile using microarray analysis of three patients with macrodactyly (M1, M2 and M3) and five healthy controls (HC1, HC2, HC3, HC4 and HC5). Red represents upregulated genes and green represents downregulated genes. The heatmap shows 165 transcripts filtered with a fold change threshold $\geq 5$, including 22 upregulated and 143 downregulated genes associated with nerve overgrowth. (B) GO term analysis of differentially expressed genes in macrodactyly. Expression data were obtained from microarray analysis of transcripts with $\mathrm{s} \geq 5$-fold expression change and $\mathrm{P}<0.05$, and grouped into GO terms. The pie chart shows that $27.5 \%$ of all GO terms identified were broadly grouped into metabolic process, $20.1 \%$ were involved in the response to the stimulus and $16.9 \%$ were involved in immune system process. GO, Gene Ontology.

target genes were further confirmed at the transcriptional level. The results showed that, of the six candidate genes, VIP, FXYD3, GRIN3A and MAPT were upregulated, which was consistent with the microarray data (Fig. 2A). To further confirm the upregulation of the above genes, retinoic acid was used to induce the neuronal differentiation of SH-SY5Y cells (Fig. 2B), and the mRNA levels of VIP, FXYD3, GRIN3A and $M A P T$ in the SH-SY5Y cells were measured using RT-qPCR analysis. As presented in Fig. 2C and D, the transcriptional expressions of GRIN3A and MAPT were increased along with the induction. Subsequently, the translational levels of these two genes (Fig. 2E and F) were determined and the results also confirmed the result that GRIN3A and MAPT were upregulated along with the process of nerve axon growth during differentiation of the SH-SY5Y cells.

Signaling pathways responsible for the upregulation of target genes. To investigate the signaling pathways and events, which may stimulate nerve cell proliferation, the SH-SY5Y cells were induced by retinoic acid for $72 \mathrm{~h}$. Retinoic acid has been shown to induce cell differentiation by sustained phosphorylation of ERK1/2 or cAMP response element binding protein (8-10). Therefore, PD98059 and H89 were added to the cells to inhibit the ERK1/2 and cAMP/PKA pathways, respectively. The results showed that at transcriptional and translational level, the expression of GRIN3A was regulated by the cAMP/PKA pathway, whereas the expression of MAPT was affected by the ERK1/2 pathway (Fig. 3A-D).

\section{Discussion}

Macrodactyly is an uncommon congenital nervous system disease, and is characterized by the proliferation of nerve fibers, vessels, subcutaneous fat and other tissues (1-3). The pathogenesis of macrodactyly remains to be fully elucidated, therefore, the identification of genes expressed at high levels in macrodactyly may reveal factors, which promote nerve overgrowth and contribute to the pathogenesis of this condition.

In the present study, the gene expression profiles were compared between patients with macrodactyly and healthy 
A

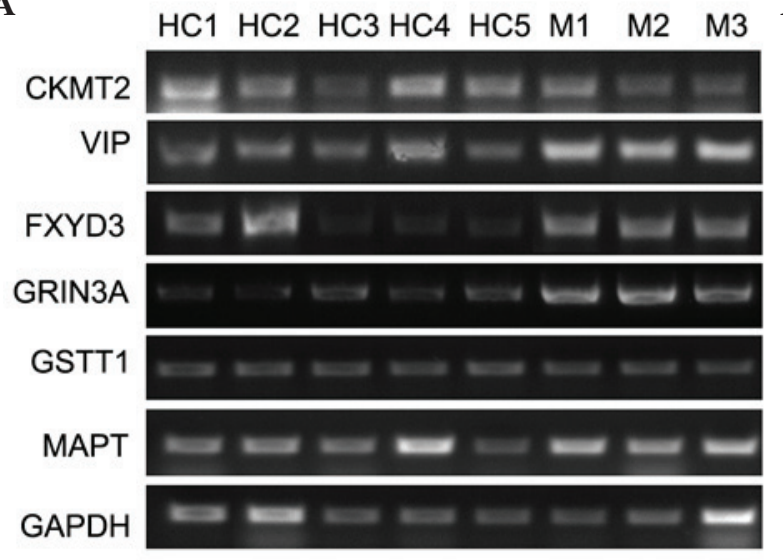

B

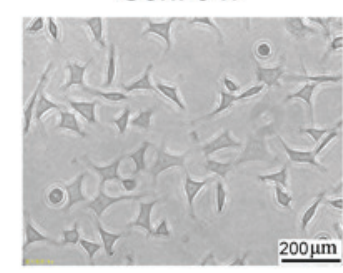

Con. $72 \mathrm{~h}$

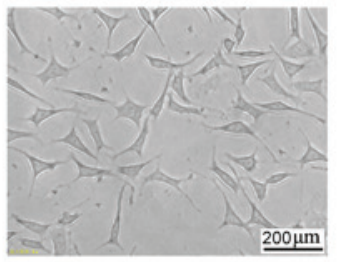

Induced $36 \mathrm{~h}$

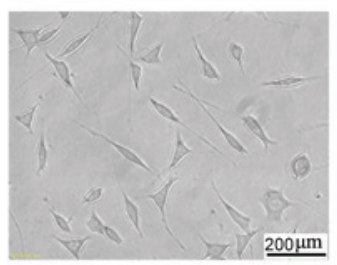

Induced $72 \mathrm{~h}$

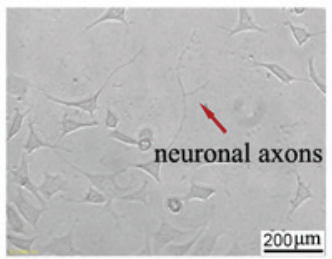

C

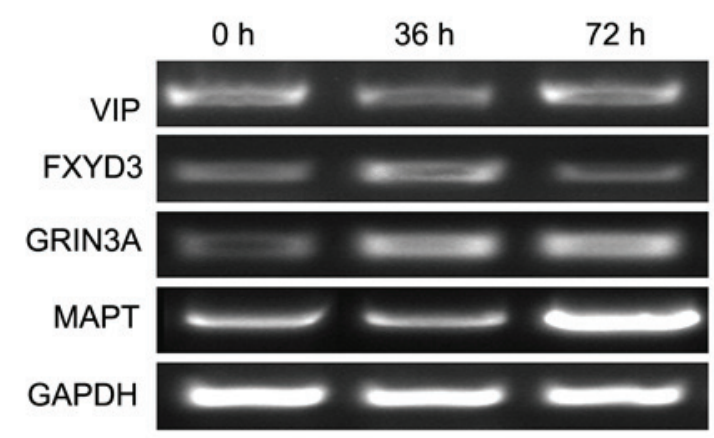

$\mathbf{E}$

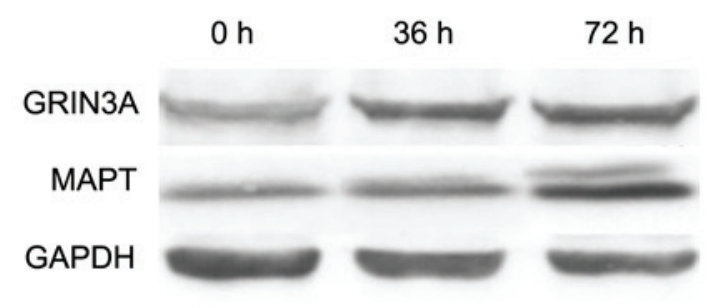

D

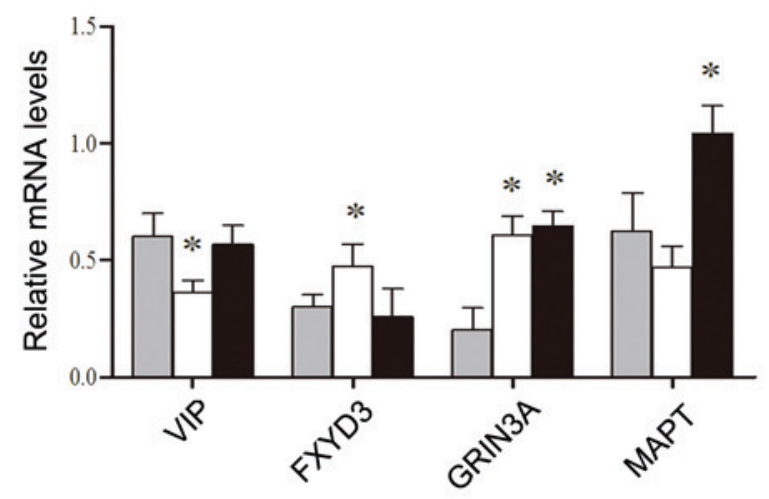

F $\square$ Con $\square$ induced $36 \mathrm{~h} \square$ induced $72 \mathrm{~h}$

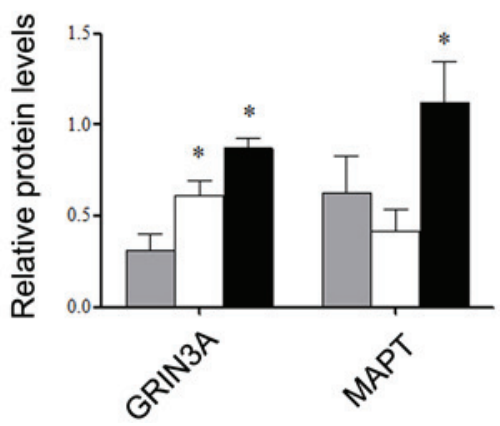

Figure 2. Analysis of mRNA levels of candidate genes associated with nerve outgrowth. mRNA levels were measured to confirm the expression profile of the six candidate genes (CKMT2, VIP, FXYD3, GRIN3A, GSTT1 and MAPT). (A) mRNA levels of CKMT2, VIP, FXYD3, GRIN3A, GSTT1 and MAPT were determined with GAPDH as the reference. (B) Differentiation of SH-SY5Y cells induced by retinoic acid for $72 \mathrm{~h}$. Scale bar=200 $\mu \mathrm{m}$. (C) Confirmation of selected genes following cell differentiation. (D) Histogram showing results of the analysis of relative mRNA levels of target genes. (E) Western blot analysis of translational levels following differentiation. (F) Quantification of western blotting. "P $<0.05$, vs. Con. CKMT2, creatine kinase, mitochondrial 2; VIP, vasoactive intestinal peptide; FXYD3, FXYD domain-containing ion transport regulator 3; GRIN3A, glutamate ionotropic receptor NMDA 3A; GSTT1, glutathione S-transferase $\theta 1 ; M A P T$, microtubule-associated protein tau; Con, control.

controls. It was found that a significant number of genes (143; $87 \%$ ) were downregulated ( $\geq 5$-fold), whereas only $22(13 \%)$ genes were upregulated ( $\geq 5$-fold). Among the downregulated genes, apoptosis-associated factors, including B cell lymphoma 2, cell division cycle $14 \mathrm{~B}$ and pentraxin 3 , and microtubule formation associated promoters, including $M A P 1, M A P T$ and sorting nexin 22, were downregulated, as expected. Of note, pro-inflammatory cytokines or associated receptors, including interleukin $(I L) 1 \beta, I L 1$ receptor $(R), I L 7 R$ and $I L 8$, and the regulators of cell-cell or cell-extracellular matrix interactions, including chemokine ( $\mathrm{C}-\mathrm{C}$ motif) ligand $20, \mathrm{C}-\mathrm{X}-\mathrm{C}$ chemokine receptor type 4 , integrin $\alpha \mathrm{X}$, integrin $\beta 8$, major facilitator superfamily domain-containing 2 , plasminogen activator, urokinase and selectin $\mathrm{L}$, were significantly downregulated. It is known that inflammatory cytokines and cell adhesion molecules are frequently overexpressed in human cancer, and are involved in oncogenesis and metastasis by improving extracellular matrix degradation (11-15). The results of the present study indicated that cell invasion and metastasis may be restrained in macrodactyly, 
A

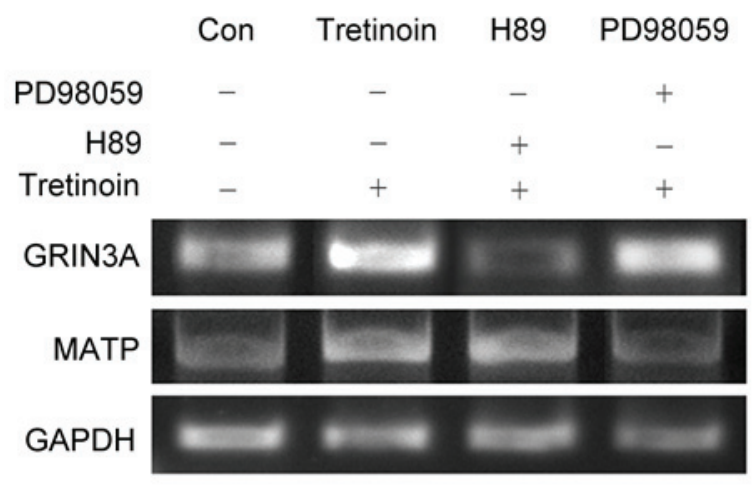

C

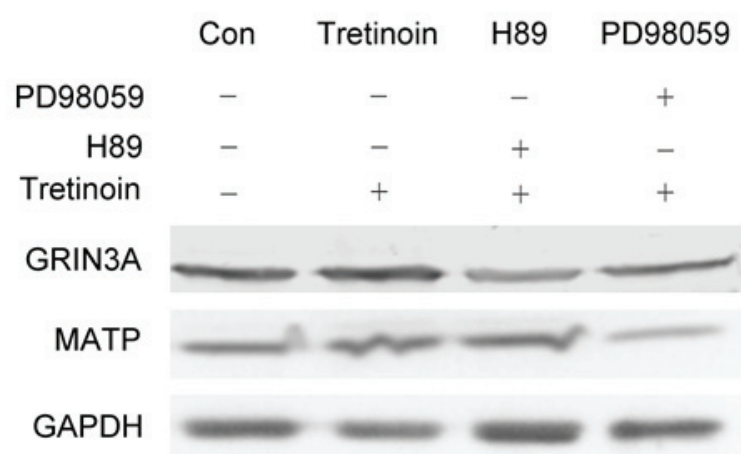

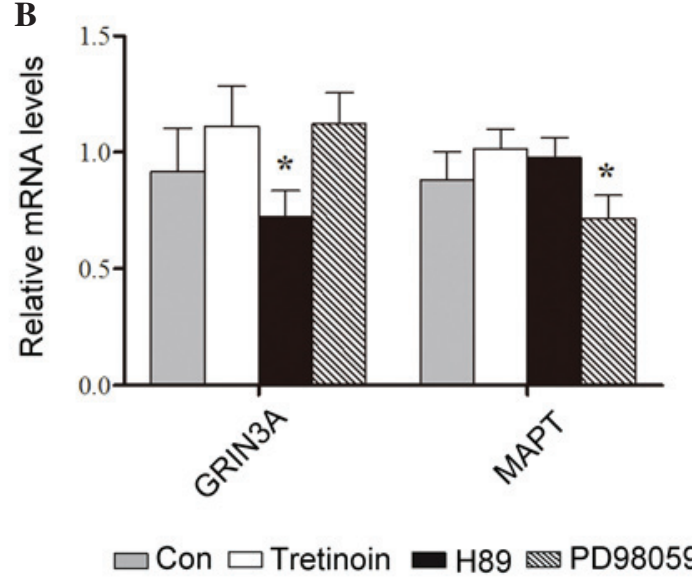

D

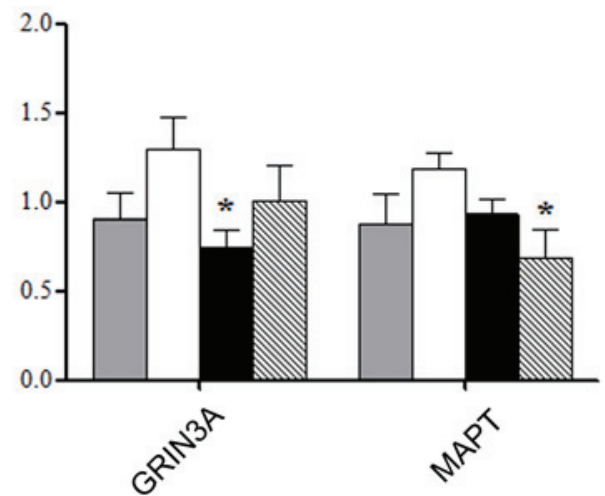

Figure 3. Signaling pathways relevant to nerve overgrowth. SH-SY5Y cells were induced by retinoic acid, and treated with PD98059 and H89 to inhibit extracellular signal-regulated kinase $1 / 2$ and cAMP/protein kinase A pathways, respectively. Expression levels of GRIN3A and MAPT were detected using (A and B) reverse transcription-quantitative polymerase chain reaction and (C and D) western blotting analyses. ${ }^{*} \mathrm{P}<0.05$, vs. retinoic acid (tretinoin) and Con groups. GRIN3A, glutamate ionotropic receptor NMDA 3A; MAPT, microtubule-associated protein tau; Con, control.

and this may explain why macrodactyly seldom develops to malignancy.

In addition, the present study found that angiogenesis-associated positive regulators, including vascular endothelial growth factor $(V E G F) A / B / C$, Notch, $\delta$-like 4 and macrophage migration inhibitory factor, were not expressed at high levels, and a number even showed decreased expression, including fibroblast growth factor receptor 1, microRNA 2 (mir21) and mir221. It is well known that vessel overgrowth is common in tumors, particularly in malignancy $(16,17)$. Therefore, the present study hypothesized that the abnormal vascular proliferation observed in macrodactyly is not due to hyperplastic nerve tissue directly.

The genes, which identified as being upregulated in the patients with macrodactyly were considered to be involved in promoting nerve overgrowth. The present study found 14 downregulated ( $>10$-fold) genes and six upregulated $(>10$-fold) genes (CKMT2, VIP, FXYD3, GRIN3A, GSTT1 and MAPT) in the macrodactyly samples. Subsequently, the upregulated levels of VIP, FXYD3, GRIN3A and MAPT were confirmed using RT-qPCR analysis. GRIN3A and MAPT have been shown to be involved in stimulating nerve growth. $M A P T$, is involved in improving nerve proliferation, and is expressed at high levels in multiple neurodegenerative disorders, including progressive supranuclear palsy, Parkinson's disease and Alzheimer's disease $(18,19)$. As an ionotropic glutamate receptor, GRIN3A is involved in regulating nerve signal transduction $(20,21)$.
Nerve growth is a result of the combined effects of multiple genes and signaling networks, thus selecting positive regulators from a nerve outgrowth model may be a promising strategy for identifying key factors and signaling pathways. To investigate the signaling pathways responsible for promoting nerve growth, retinoic acid was used to induce the differentiation of SH-SY5Y cells (7). By inhibiting the ERK1/2 pathway and cAMP-PKA pathway, which are involved in neurite proliferation, it was found that GRIN3A showed a significant correlation with the cAMP-PKA pathway, whereas $M A P T$ was affected by the ERK1/2 pathway.

Increased expression levels of MAPT and GRIN3A may lead to the abnormal pathologic condition of nerve tissue in macrodactyly and, to a certain extent, may have potential applications in nerve regeneration. It is known that axon continuity is always interrupted during nerve damage, and the distal axonal tract finally undergoes degeneration. Thus, identifying techniques to reconstruct the structure of nerve tissue, and restore its function following nerve injury and repair has been one of the key areas of investigation in tissue engineering. Gene therapy is one potential approach in the treatment of traumatic nerve injury. Various genes are being considered as candidates for promoting nerve regeneration, including VEGF $(22,23)$. Based on the findings from the present study, MAPT and GRIN3A warrant further examination as two potential factors, which may contribute to nerve regeneration and reconstruction following nerve injury. 


\section{Acknowledgements}

The authors would like to thank Medjaden Bioscience Limited (Hong Kong, China) for assistance with proofreading.

This study was supported by grants from the National Natural Science Foundation of China (grant nos. 30972610 and 81273240), the Health Department Research Projects in Jilin Province (grant no. 2009Z054) and the Norman Bethune Program of Jilin University (grant no. 2012206).

\section{References}

1. Ho CA, Herring JA and Ezaki M: Long-term follow-up of progressive macrodystrophia lipomatosa. A report of two cases. J Bone Joint Surg Am 89: 1097-1102, 2007.

2. Lau FH, Xia F, Kaplan A, Cerrato F, Greene AK, Taghinia A, Cowan CA and Labow BI: Expression analysis of macrodactyly identifies pleiotrophin upregulation. PLoS One 7: e40423, 2012.

3. Biesecker LG, Aase JM, Clericuzio C, Gurrieri F, Temple IK and Toriello H: Elements of morphology: Standard terminology for the hands and feet. Am J Med Genet A 149A: 93-127, 2009.

4. Rios JJ, Paria N, Burns DK, Israel BA, Cornelia R, Wise CA and Ezaki M: Somatic gain-of-function mutations in PIK3CA in patients with macrodactyly. Hum Mol Genet 22: 444-451, 2013.

5. Rohilla S, Jain N,Sharma R and Dhaulakhandi DB: Macrodystrophia lipomatosa involving multiple nerves. J Orthop Traumatol 13: 41-45, 2012.

6. Livak KJ and Schmittgen TD: Analysis of relative gene expression data using real-time quantitative PCR and the 2(-Delta Delta C(T)) method. Methods 25: 402-408, 2001.

7. Cheung YT, Lau WK, Yu MS, Lai CS, Yeung SC, So KF and Chang RC: Effects of all-trans-retinoic acid on human SH-SY5Y neuroblastoma as in vitro model in neurotoxicity research. Neurotoxicology 30: 127-135, 2009.

8. Huang Y, Boskovic G and Niles RM: Retinoic acid-induced AP-1 transcriptional activity regulates B16 mouse melanoma growth inhibition and differentiation. J Cell Physiol 194: 162-170, 2003.

9. Hung SP, Hsu JR, Lo CP, Huang HJ, Wang JP and Chen ST: Genistein-induced neuronal differentiation is associated with activation of extracellular signal-regulated kinases and upregulation of p21 and N-cadherin. J Cell Biochem 96:1061-1070, 2005.

10. Tegenge MA, Roloff F and Bicker G: Rapid differentiation of human embryonal carcinoma stem cells (NT2) into neurons for neurite outgrowth analysis. Cell Mol Neurobiol 31: 635-643, 2011.
11. Bachireddy P, Rakhra K and Felsher DW: Immunology in the clinic review series; focus on cancer: Multiple roles for the immune system in oncogene addiction. Clin Exp Immunol 167: 188-194, 2012.

12. Schreiber RD, Old LJ and Smyth MJ: Cancer immunoediting: Integrating immunity's roles in cancer suppression and promotion. Science 331: 1565-1570, 2011.

13. Mantovani A, Romero P, Palucka AK and Marincola FM: Tumour immunity: Effector response to tumour and role of the microenvironment. Lancet 371: 771-783, 2008.

14. Moh MC and Shen S: The roles of cell adhesion molecules in tumor suppression and cell migration: A new paradox. Cell Adh Migr 3: 334-336, 2009.

15. Nair KS, Naidoo R and Chetty R: Expression of cell adhesion molecules in oesophageal carcinoma and its prognostic value. J Clin Pathol 58: 343-351, 2005.

16. Hedlund EM, Hosaka K, Zhong Z, Cao R and Cao Y: Malignant cell-derived PIGF promotes normalization and remodeling of the tumor vasculature. Proc Natl Acad Sci USA 13: 17505-17510, 2009.

17. Carmeliet P: Angiogenesis in life, disease and medicine. Nature 438: 932-936, 2005.

18. Elbaz A, Ross OA, Ioannidis JP, Soto-Ortolaza AI, Moisan F, Aasly J, Annesi G, Bozi M, Brighina L, Chartier-Harlin MC, et al: Independent and joint effects of the MAPT and SNCA genes in Parkinson's disease. Ann Neurol 69: 778-792, 2011.

19. Caffrey TM and Wade-Martins R: Functional MAPT haplotypes: Bridging the gap between genotype and neuropathology. Neurobiol Dis 27: 1-10, 2007.

20. Liu XJ and Salter MW: Glutamate receptor phosphorylation and trafficking in pain plasticity in spinal cord dorsal horn. Eur J Neurosci 32: 278-289, 2010.

21. Oh MC, Kim JM, Safaee M, Kaur G, Sun MZ, Kaur R, Celli A, Mauro TM and Parsa AT: Overexpression of calcium-permeable glutamate receptors in glioblastoma derived brain tumor initiating cells. PLoS One 7: e47846, 2012.

22. Wong WK, Cheung AW, Yu SW, Sha O and Cho EY: Hepatocyte growth factor promotes long-term survival and axonal regeneration of retinal ganglion cells after optic nerve injury: Comparison with CNTF and BDNF. CNS Neurosci Ther 20: 916-929, 2014.

23. Pelletier J, Roudier E, Abraham P, Fromy B, Saumet JL, Birot O and Sigaudo-Roussel D: VEGF-A promotes both proangiogenic and neurotrophic capacities for nerve recovery after compressive neuropathy in rats. Mol Neurobiol 51: 240-251, 2015. 